\title{
Sloppy Size Control of the Cell Division Cycle
}

\author{
JOHN J. TYSON \\ Department of Biology, Virginia Polytechnic Institute and State University, \\ Blacksburg, VA 24061, U.S.A. \\ AND \\ Odo DIEKManN
}

Centrum voor Wiskunde en Informatica, Kruislaan 413, NL-1098 SJ Amsterdam, The Netherlands

(Received 15 April 1985, and in revised form 14 August 1985)

In an asynchronous, exponentially proliferating cell culture there is a great deal of variability among individual cells in size at birth, size at division and generation time (=age at division). To account for this variability we assume that individual cells grow according to some given growth law and that, after reaching a minimum size, they divide with a certain probability (per unit time) which increases with increasing cell size. This model is called sloppy size control because cell division is assumed to be a random process with size-dependent probability. We derive general equations for the distribution of cell size at division, the distribution of generation time, and the correlations between generation times of closely related cells. Our theoretical reqults are compared in detail with experimental results (obtained by Miyata and coworkers) for cell division in fission yeast, Schizosaccharomyces pombe. The agreement between theory and experiment is superior to that found for any other simple models of the coordination of cell growth and division.

\section{Introduction}

Many types of cells, including bacteria, unicellular eukaryotic organisms, and cells isolated from higher plants and animals, can be grown in laboratory cultures. Under appropriate nutritional conditions such populations of cells proliferate exponentially; that is, cell number and total cell mass increase exponentially at the same specific growth rate. Though the macroscopic properties of the culture as a whole follow a deterministic growth law, the descriptors of individual cells (such as size at division and age at division) are highly variable. For instance, the coefficient of variation ( $\mathrm{CV}=$ standard deviation/mean) of size at division is typically $10 \%$ and the CV of age at division is typically $20 \%$ or more.

The variabilities of size and age at division found in experiments can be described by histograms like those in Fig. 1. For many years theoreticians have tried to understand generation time histograms in terms of age-dependent probabilistic models of progress through the cell cycle (Rahn, 1932; Kendall, 1948; Lebovitz \& Rubinow, 1969; Smith \& Martin, 1973; Brooks et al., 1980). In these models the 


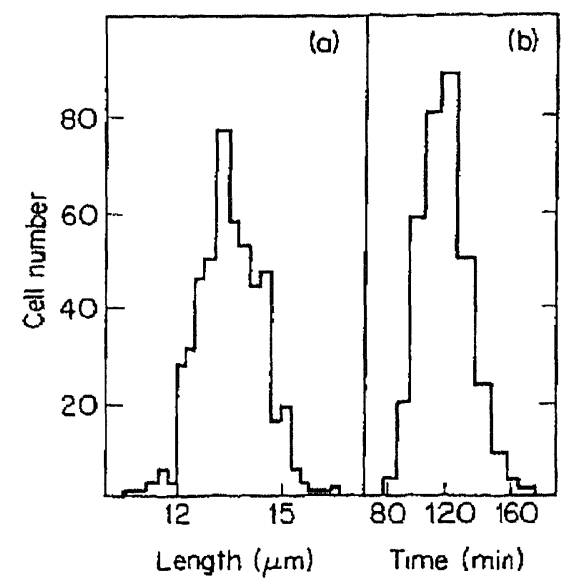

FrG. 1. Histograms of the length of cells with cell plates (a) and generation time (b) in an asynchronous, exponentially growing population of fission yeast, Schizosaccharomyces pombe (Miyata et al., 1978; used by permission of the authors and the Japan Society for Cell Biology). For the cell length distribution, mean $=13.4 \mu \mathrm{m}$ and $\mathrm{CV}=7.5 \%$; for the generation time distribution, mean $=116 \mathrm{~min}$ and $\mathrm{CV}=13.8 \%$.

probability that a cell will divide in the next brief interval of time is taken to be a function of the cell's present age, independent of cell size. Such models ignore the far-ranging evidence that cell size plays an important role in determining when cells divide (Mitchison, 1977; John, 1981; Nurse \& Streiblova, 1984). On the other hand, size control models discussed in the literature (e.g. Fantes et al., 1975) are, for the most part, deterministic models which ignore the evident variability in progress through the cell cycle.

Koch \& Schaechter (1962) were first to incorporate probabilistic effects into a deterministic model for cell growth and size-controlled division. In their model the probability that a cell will divide in the next brief interval of time is dependent on the cell's present size, not on its present age or its size at birth. Their model was based on four assumptions: (i) each individual cell grows exponentially with a specific growth rate the same as that for the culture as a whole; (ii) on the average, a cell grows until it reaches a critical size (under physiological control) and then divides; (iii) whereas the average size of a cell at division is strictly controlled, the actual size at division of individual cells may vary slightly due to random events; (iv) mother cells divide into daughters of equal size. Koch \& Schaechter were interested in the implications of these assumptions regarding cell size distributions, variability in generation times, and correlations of generation times between sister cells and mother-daughter pairs. The conclusions reached by Koch \& Schaechter were, for the most part, correct, but they were given firm mathematical demonstration only later by Powell (1964). Among other things, Powell pointed out that Koch \& Schaechter's assumptions (ii) and (iii) are better stated as: (ii') there is a critical size $(X)$ below which cells are incapable of dividing, (iii') cells larger than the critical size have a probability of division that increases with increasing size such that no cell ever exceeds size $2 X$ before dividing. Powell also showed how to relax assumption (iv) so that sister cells might have different sizes at birth.

Sadly, the work of Koch, Schaechter and Powell has been neglected for many years. Only recently have experimentalists become interested again in size-dependent 
probabilistic models of progress through the cell cycle (Nurse, 1980; Lord \& Wheals, 1981; Wheals, 1982) and have theoreticians begun again to investigate the consequences of such models (Diekmann et al,, 1983; Lasota \& Mackey, 1984; Tyson \& Hannsgen, 1985). In this paper we consider models of the sort pioneered by Koch, Schaechter and Powell, which we call "sloppy size control" models, after the suggestion of Wheals (1982).

In section 2 we present expressions for the probability distributions for division size and generation time. (These formulae are derived in Appendix A.) In section 3 we compare our theoretical distributions with experimental histograms obtained by Miyata et al., (1978) for the fission yeast Schizosaccharomyces pombe. In section 4 we present equations for the so-called "beta curve" and for the correlation coefficients for generation times of sister cell pairs and mother-daughter pairs (the formulae are derived in Appendix B), and we compare these results with Miyata's data on fission yeast cells. In section 5 we investigate the differences between exponential and logistic growth of individual cells.

Before proceeding with the derivations we must introduce some definitions and conventions.

\section{Distributions, Densities and Samples}

Experimental histograms, such as those in Fig. 1, are presumably manifestations of underlying continuous probability density functions. That is, we can define two continuous functions $\phi(x)$ and $f(T)$ such that

$$
\begin{aligned}
& \text { the number of cells with size } \\
& \text { at division between } x \text { and } x+\mathrm{d} x
\end{aligned}=N \phi(x) \mathrm{d} x+\mathrm{o}(\mathrm{d} x)
$$

and

$$
\begin{aligned}
& \text { the number of cells with generation }=N f(T) \mathrm{d} T+\mathrm{o}(\mathrm{d} T) \\
& \text { time between } T \text { and } T+\mathrm{d} T
\end{aligned}
$$

where $N$ is the total number of cells in the sample, and $o(\cdot)$ denotes correction terms that are negligible for sufficiently small argument. To be complete we must specify the sample over which the distributions are defined. There are two different sampling conventions that are routinely used.

When sampling contemporaneously, one considers all cells that are alive during a certain brief time interval (between $t$ and $t+\mathrm{d} t$ ). Those cells which divide in this time interval provide a convenient sample for $\phi(x)$ and those which are born in this interval provide a convenient sample for $f(T)$. Notice that $\phi(x)$ is in general different from the density function for size at division defined on a sample newborn cells; the latter density function is denoted $\phi_{b}(x)$ ( $b$ for "babies"). Similarly $f(T)$ is in general different from the density function for generation time defined on a sample of dividing cells; the latter density function is denoted $f_{m}(T)$ ( $m$ for "mothers"). We are using the notions and notations for contemporaneous sampling that were introduced by Painter \& Marr (1968). Notice that the density functions depend, in general, on the time $t$ at which the samples are taken. However, after 
establishment, cultures quickly reach a steady state of growth and division, in which the distributions of age and size no longer depend on time of sampling. $t$

An alternative procedure is to sample cells by generations. In this case one considers the sample of all cells that belong to a certain generation of descendants of a given initial cell. After 10 generations, say, there would be 1024 cells in this sample (assuming no cell death). In this convention $\phi(x)$ and $f(T)$ are defined over the sample of all cells belonging to generation $n$, for sufficiently large $n$ so that the distributions have reached the steady state.

When dealing with contemporaneous samples we shall use the notation $\phi(x)$ and $f(T)$ for the steady-state probability density functions, whereas by $\phi_{*}(x)$ and $f_{*}(T)$ we shall denote the analogous functions defined over generation-based samples. Where necessary we shall use capital letters for cumulative distribution functions; e.g.

$$
\Phi(x)=\int_{0}^{x} \phi(\xi) \mathrm{d} \xi, \quad F(t)=\int_{0}^{t} f(s) \mathrm{d} s .
$$

We shall use the word "distribution" to refer loosely to either probability density functions or cumulative distribution functions.

\section{Size and Generation Time Distributions}

To define a sloppy size control model, we must specify a growth law for individual cells,

$$
\mathrm{d} x / \mathrm{d} t=V(x), x(t)=\text { cell size at time } t,
$$

and a division probability function,

$$
\begin{aligned}
b(x) \mathrm{d} t= & \text { probability that a cell of size } x \text { at time } t \text { will } \\
& \text { divide in the time interval }(t, t+\mathrm{d} t) .
\end{aligned}
$$

We take $V(x)$ and $b(x)$ as given functions. We shall assume that there is a minimum size at division $(x=a)$ and a maximum size at division $(x=1)$, and that

$$
\frac{1}{2}<a<1 \text {. }
$$

We assume that $a>\frac{1}{2}$ for several reasons. First, if $a>\frac{1}{2}$, then all cells progress through the size range $\left[\frac{1}{2}, a\right]$ at some time in their life, and this situation greatly simplifies the derivation of steady state size distributions (Powell, 1964). If $a<\frac{1}{2}$, then those cells born with size $>a$ would have a finite probability of dividing immediately after birth, which is certainly unrealistic. To admit $a<\frac{1}{2}$, we would

†In dynamical models of cell growth and division one can show theoretically that, as a rule, cell size distributions converge towards stable ("steady state") distributions. Ironically, it turns out that the combination of exponential cell growth and exactly symmetric division forms the only exception to this rule (Bell \& Anderson, 1967; Diekmann et al., 1984; also Chapters I and II of Metz \& Diekmann, in preparation). Nevertheless we will base most of our calculations in what follows on precisely these assumptions of exponential growth and exactly symmetric division. Our rationale is that any small aberration from either true exponential growth or exactly symmetric division yields stable distributions which are close to the ones we calculate. We refer to Heijmans (1984) for relevant results concerning asymmetric division. 
have to modify the model to include a minimum generation time, and then the probability of cell division would become a function of cell age as well as cell size. Such complications we prefer to avoid, unless they are forced on us by experimental facts. Our assumption that $a>\frac{1}{2}$ implies that the maximum size at birth $\left(x=\frac{1}{2}\right)$ is less than the minimum size at division $(x=a)$; i.e. that the birth size distribution and the division size distribution do not overlap. There is experimental evidence that such lack of overlap is true for many cell types (Powell, 1964).

As long as $a>\frac{1}{2}$ there is an absolute minimum generation time given by the time needed to grow from the maximum size at birth to the minimum size at division:

$$
T_{\min }=\int_{1 / 2}^{a}[V(x)]^{-1} \mathrm{~d} x .
$$

The observed smallest generation time may be considerably larger than $T_{\min }$, however, because the probability density function for generation time, as we shall see, stays very close to zero for times considerably larger than $T_{\operatorname{mir}}$. For our purposes here, it is not necessary to introduce any other contribution to a minimum generation time (e.g. a minimum duration of $G_{1}+S+G_{2}+M$, the classical phases of the cell cycle). In other circumstances, for instance, in describing the recovery of oversized cells to normal size at division (Fantes et al., 1975; Sudbury \& Grant, 1975), it may be necessary to include a minimum duration of $G_{1}+S+G_{2}+M$.

A maximum size at division might come about in either of two ways. If cell growth is unlimited, then the probability of cell division must become infinitely large as cell size approaches the maximum size at division. This possibility is considered in section 3 where we assume exponential growth, $V(x)=k x$, and singular behaviour of the division probability function, $b(x)$, as $x \rightarrow 1$. The second possibility, pursued in section 5 , is that growth itself is limited, so that no cell ever grows larger than $x=1$. In this case the division probability function need not be singular at $x=1$.

In order to examine the concept of sloppy size control in light of the experimental histograms in Fig. 1, we must derive the predictions of the model concerning the probability density functions for cell size at division and cell age at division. These derivations are carried out in Appendix A, and we report only the results here.

First let us define

$$
\begin{aligned}
& E(x)=\exp \left\{-\int_{a}^{x}[b(\xi) / V(\xi)] \mathrm{d} \xi\right\}, \\
& G(x)=\int_{a / 2}^{x}[V(\xi)]^{-1} \mathrm{~d} \xi .
\end{aligned}
$$

$E(x)$ is the probability that a cell passing size $a$ will reach size $x$ before dividing. By assumption $E(1)=0 . G(x)$ is the time needed for a cell to grow from size $a / 2$ (the minimum size at birth) to size $x$. In these terms we have for the distribution of division sizes in a contemporaneous sample of dividing cells

$$
\phi(x)=b(x) \mathrm{e}^{-k \sigma(x)} E(x) / V(x), \quad a<x<1,
$$

where $k$ is the specific growth rate of the population as a whole, and where, in general, the right-hand-side of (1) should be multiplied by a constant so that 
$\int_{a}^{1} \phi(x) \mathrm{d} x=1$. If the dividing cells all belong to the same generation, then

$$
\phi_{*}(x)=b(x) E(x) / V(x), \quad a<x<1 .
$$

We also need to know the distribution of division sizes in a contemporaneous sample of newborn cells

$$
\phi_{b}(x)=b(x) E(x) / V(x), \quad a<x<1 .
$$

In general, $\phi_{b}$ and $\phi_{*}$ need not be identical. That they are so here is a special consequence of our assumption that $a>\frac{1}{2}$.

Knowing these size distributions, we can now write integral expressions for the distribution of generation times. For a contemporaneous sample of newborn cells,

$$
f(T)=\int_{a / 2}^{1 / 2} 2 \phi(2 x) \phi_{b}(m(x, T)) V(m(x, T)) d x, \quad T_{\min }<T<T_{\max },
$$

where

$$
\begin{aligned}
T_{\min } & =G(a)-G\left(\frac{1}{2}\right), \\
T_{\max } & =G(1),
\end{aligned}
$$

and $m(x, t)$ is the size of a cell at time $t$ if its size $=x$ at time 0 . For a sample of cells all belonging to the same generation,

$$
f_{*}(T)=\int_{a / 2}^{1 / 2} 2 \phi_{*}(2 x) \phi_{*}(m(x, T)) V(m(x, T)) \mathrm{d} x, \quad T_{\min }<T<T_{\max }
$$

\section{Application to Fission Yeast}

To compare our theoretical equations for division-size and generation-time distributions to the experimental histograms in Fig. 1, we must specify a growth law $V(x)$ and a division probability $b(x)$ suitable for fission yeast cultures. But first we must be more specific about what we mean by "size". In the sloppy size control model we have in mind by cell size some attribute of the cell which increases continuously as the cell grows, whose increase is not directly linked to the cell division cycle (DNA synthesis, nuclear division, cell division), whose level can be monitored biochemically by the cell, and which, on reaching a certain level, can trigger cell division and a new round of DNA synthesis. The attribute might be cell volume, dry mass, protein content, or some specific division-activating factor. In the absence of any direct information about the identity of the size variable, we can reasonably associate cell size with the easily measured variable, cell length. Since fission yeast cells grow only by elongation, cell length is proportional to cell volume. Since mother cells divide into equally sized daughters, our assumption of symmetric division is warranted.

Having settled on cell length as our size variable, we next ask what is the growth law for cell length in fission yeast. There is no clear cut experimental answer to this question. Mitchison \& Nurse (1985) report that cell length increases in a complicated fashion: during the first $50 \%$ of the cycle, length increases linearly; for the next 
$25 \%$ of the cycle, length continues to increase linearly but at a greater rate; and for the last $25 \%$ of the cycle, while a new septum is being formed, there is no increase in cell length. At the same time, according to Mitchison (1957), dry mass increases linearly throughout the cycle, so there must be a fluctuation in cell bouyant density. On the other hand, Kubitschek \& Ward (1985) report that the bouyant density of $S$. pombe cells is constant during the cell cycle, and Kubitschek (1985) reports that cell length increases linearly at constant rate throughout the cell cycle. (Kubitschek sees a plateau in cell length measurements for the last $25 \%$ of the cell cycle only under growth conditions that he considers perturbed.) To make matters worse, measurements of the rate of protein synthesis during the fission yeast cell cycle, by incorporation of pulses of tritiated amino acids (Creanor \& Mitchison, 1982), are not consistent with any of the reported patterns of cell length or dry mass increase.

There seems to be no compelling reason to choose any one growth law over another, so we intend to investigate several. Though exponential growth is one possibility that is never seen experimentally for fission yeast cells, we shall assume, in this section, that cell size (length) increases exponentially. We treat the case of exponential growth for several reasons: $(1)$ it is the easiest case from a theoretical point-of-view, (2) exponential growth seems to be the rule for many cell types other than $S$. pombe, and (3) the assumption of exponential growth is very successful in accounting for the data in Fig. 1, as we shall see. In section 5 we shall investigate the consequences of logistic growth, and in a later publication we intend to study strictly linear growth and linear growth with a plateau.

So, for the present, we assume that individual cells grow exponentially, $V(x)=k x$.

The division probability function is unknown experimentally but it must satisfy the following mathematical restrictions in order that our theory apply

$$
b(x)=0, \quad 0<x<a,
$$

and

$$
\lim _{x \uparrow 1} \int_{a}^{x}[b(\xi) / V(\xi)] \mathrm{d} \xi=+\infty
$$

A convenient function satisfying these requirements is

$$
b(x)= \begin{cases}0, & 0<x<a, \\ \hat{b}(x-a)^{2} /(1-x), & a<x<1, \quad \hat{b}=\text { positive constant } \\ 0, & x \geqslant 1 .\end{cases}
$$

With these choices for $b(x)$ and $V(x)$ we find that

$$
G(x)=k^{-1} \ln (2 x / a), \quad m(x, t)=x \mathrm{e}^{k t},
$$

and

$$
E(x)=\exp \left\{\beta(x-a)-a^{2} \beta \ln (x / a)+(1-a)^{2} \beta \ln [(1-x) /(1-a)]\right\}
$$

where $\beta=\hat{b} / k$. From equations (2) and (3) we have

$$
\phi_{b}(x)=\phi_{*}(x)=[\beta / a(1-a)](x-a)^{2} \mathrm{e}^{\beta(x-a)}(x / a)^{-a^{2} \beta-1}[(1-x) /(1-a)]^{(1-a)^{2} \beta-1}
$$


From equation (1) we have, except for a normalization factor,

$$
\phi(x)=(x-a)^{2} \mathrm{e}^{\beta(x-a)} x^{-a^{2} \beta-2}(1-x)^{(1-a)^{2} \beta-1}, \quad a<x<1 .
$$

There are only two arbitrary parameters in this function: $a$, the ratio of minimum to maximum division size, and $\beta$, the ratio of $\hat{b}$ to $k$. The generation time distribution, given by equation (4), is

$$
f(T)=\left\{\begin{array}{l}
k\langle x\rangle_{H} \mathrm{e}^{k T} \int_{a e^{-k T}}^{1 / 2} \phi_{b}(2 x) \phi_{b}\left(x e^{k T}\right) \mathrm{d} x, \ln (2 a) \leq k T \leq \ln 2 \\
k\langle x\rangle_{H} \mathrm{e}^{k T} \int_{a / 2}^{\mathrm{e}^{-k T}} \phi_{b}(2 x) \phi_{b}\left(x \mathrm{e}^{k T}\right) \mathrm{d} x, \ln 2 \leq k T \leq \ln (2 / a)
\end{array}\right.
$$

where $\phi_{b}(\cdot)$ is given by (7) and $\langle x\rangle_{H}$ is the harmonic mean of $\phi_{b}$

$$
\frac{1}{\langle x\rangle_{H}}=\int_{a}^{1} x^{-1} \phi_{b}(x) \mathrm{d} x \text {. }
$$

In Fig. 2 are plotted the yeast cell histograms in comparison with functions (8) and (9) for the parameter values $a=0 \cdot 6, \beta=100$. Besides $a$ and $\beta$ there are three other parameters at our disposal: two normalization constants which must be chosen to convert probability density into "number of cells", and a scaling constant to convert $x$ into "cell length". The specific growth rate $k$ is not a parameter at our disposal: from the experimentally determined mean generation time we find that $k=0.693 / 116 \mathrm{~min} \simeq 0.006 \mathrm{~min}^{-1}$.

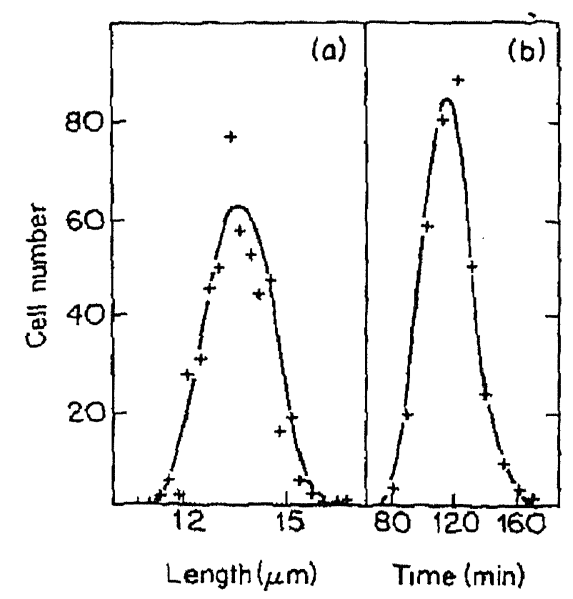

FIG. 2. Probability density functions for division size (a) and generation time (b), predicted by equations ( 8$)$ and $(9)$ and compared with the fission yeast data $(t)$ from Fig. 1. Parameter values: $a=0.6, \beta=100$ (normalization constants were adjusted to give comparable overall numbers of cells).

Notice that the smallest observed generation time (approx. $75 \mathrm{~min}$ ) is considerably larger than the theoretically absolute minimum generation time, $T_{\min }=k^{-1} \ln (2 a)=$ $30 \mathrm{~min}$, even though the predicted density function fits the observed histogram quite well even for small generation times. There is no evidence, from this sample of several hundred fission yeast cells, that the model is predicting too many cells with small generation times. Thus, there is no need, at present, to incorporate a minimum 
generation time longer than the time needed to grow from size $\frac{1}{2}$ to size $a$. If a larger sample of cells showed conclusively that generation time cannot be pushed as low as $30 \mathrm{~min}$, then we would have to modify our model to include a minimum time for completion of certain events of the DNA/division cycle $\left(G_{1}+S+G_{2}+M\right)$.

The good agreement between theory and experiment for both the division size distribution and the generation time distribution provides startling corroboration of the sloppy size control model. No other simple model of cell growth and division fits both distribution functions so well (Tyson, 1985).

\section{Generation Time Correlations}

Our theoretical description of cell growth and division can be tested further by examining correlations of generation times between related cells. Such correlations may be expressed in terms of product moment correlation coefficients. For dealing with sister cell correlations, Minor \& Smith (1974) have pointed out the advantages of investigating the so-called beta curve

$$
\begin{aligned}
\beta(t)= & \text { probability that sister cells have a difference in } \\
& \text { generation time greater than } t .
\end{aligned}
$$

In Appendix $\mathrm{B}$ we derive expressions from $\beta(t)$ and for the mother-daughter and sister-sister correlation coefficients. For the case of exponential growth of individual cells,

$$
\begin{gathered}
\beta(t)=2 \int_{a}^{\mathrm{e}^{-k t}} \int_{r \mathrm{e}^{k t}}^{1} \phi_{b}(r) \phi_{b}(x) \mathrm{d} x \mathrm{~d} r \\
r_{s s}=\frac{1}{2}, \quad r_{m d}=-\frac{1}{2} .
\end{gathered}
$$

The double integral in (10) has been evaluated numerically for the functions $b(x)$ and $V(x)$ given in section 3 and the parameter values $a=0.6$ and $\beta=100$. The result is displayed in Fig. 3 along with the experimental $\beta$-curve for the fission yeast population described in Fig. 1. The excellent agreement between theory and experiment provides further confirmation of the sloppy size control model.

(The shape of the curve $\beta(t)$ is quite sensitive to the value of the parameter $\beta=\hat{b} / k$. The value $\beta=100$ was chosen to fit the theoretical curve to the experimental points. This is not a "best" fit in any statistical sense but only a "casual" fit in the sense that $\beta=90$ and $\beta=110$ are obviously less satisfactory than $\beta=100$.)

From generation-time data kindly provided by Professor $H$. Miyata, we have calculated $r_{s s}$ and $r_{m d}$ for the fission yeast cultures described in Figs 1 and 3 . We find that

$$
\begin{aligned}
r_{s s} & =0.50 & & (n=50, P=100 \%) \\
r_{s s} & =0.39 & & (n=36, P=45 \%) \\
r_{m d} & =-0.44 & & (n=48, P=60 \%) .
\end{aligned}
$$

Here, $P$ is the probability, as judged by Fisher's $Z$ transformation (Daniel, 1983), that the difference between the observed and expected (11) values of the correlation 


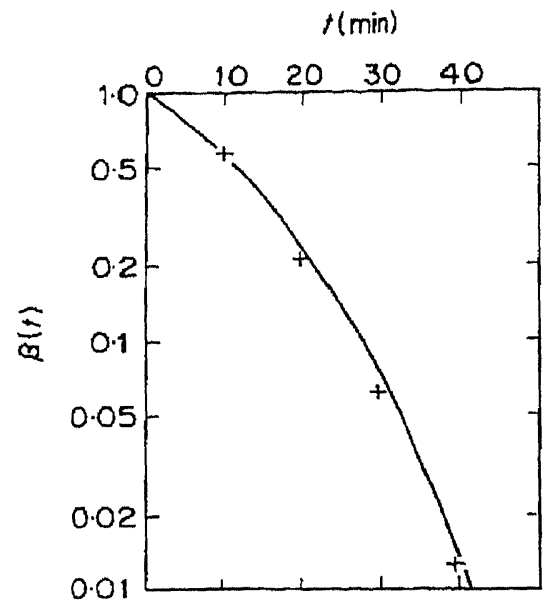

FIG. 3. Beta-curve (solid line) predicted by equation (10) and compared with data on fission yeast populations used to construct histograms in Fig. 1. Data on generation times of sister cells was kindly provided by Professor $\mathrm{H}$. Miyata. Because cells were photographed at 10 min time intervals, there is a large uncertainty in the experimental beta curve. Assuming that cell division times are scattered uniformly over these $10 \mathrm{~min}$ intervals, we calculate the points $\beta(t)$ marked by + . The $\beta$-curve given here is quite similar to the $\beta$-curve for $S$. pombe published by Fantes (1977) in that both curves have a distinct "shoulder" at small values of $t$ and an apparently exponential tail at larger values of $t$.

coefficient can be attributed to random sampling error on a sample of $n$ pairs. (The two experimental values for $r_{s s}$ refer to two different samples of sister-sister pairs.) Obviously, the measured correlation coefficients are not significantly different from the theoretical values.

\section{Logistic Growth}

In the previous sections we have based our cell cycle statistics on the assumption that individual cells grow exponentially. However, the observations of Mitchison $\&$ Nurse (1985) suggest that logistic growth may provide a more realistic description of increase in cell length in the fission yeast $S$. pombe. Therefore, in this section we investigate the effects on cell cycle statistics of assuming that

$$
V(x)=r x(1-x)
$$

Given growth law (12) we see that celis never grow larger than $x=1$, so the maximum size at division is now limited by the growth law rather than by a singularity in the division probability function. Indeed if we assume simply

$$
b(x)=\hat{b}(x-a)^{2} \text {, }
$$

then $b(x) / V(x)$ is unchanged from section 3 , except that $\beta=\hat{b} / r$ instead of $\hat{b} / k$. The specific growth rate $k$ is now given by the unique real positive root of the characteristic equation (A8), which for the functions $V(x)$ and $b(x)$ given by (12) and (13) becomes

$$
\begin{aligned}
1= & \frac{2 \beta}{a(1-a)} \int_{a}^{1}(x-a)^{2} \mathrm{e}^{\beta(x-a)}(x / a)^{-a^{2} \beta-1} \\
& \times[(1-x) /(1-a)]^{(1-a)^{2} \beta-1}[(1-x) /(2-x)]^{k / r} \mathrm{~d} x .
\end{aligned}
$$


(A good approximation for $k$ can be made by noticing that were the factor $[(1-x) /(2-x)]^{k / r}$ replaced by $\frac{1}{2}$, then (14) would be an identity because the left-hand-side of (14) would be $\int_{a}^{1} \phi_{b}(x) \mathrm{d} x$, which is necessarily unity. Roughly speaking, $(1-x) /(2-x)=(1-a) /(3-a)$ over the interval $[a, 1]$, so if we take

$$
k=r \ln (2) / \ln [(3-a) /(1-a)] \text {, }
$$

then (14) should be nearly satisfied. In our experience (15) is accurate to one significant figure. For more accuracy, (15) provides an excellent initial guess for a Newton-Raphson iterative calculation of $k$ )

Let $\kappa=k / r$. Then we can write for the division-size density function (up to a normalization factor)

$$
\phi(x)=(x-a)^{2} \mathrm{e}^{\beta(x-a)} x^{-a^{2} \beta-1-\kappa}(1-x)^{(1-a)^{2} \beta-1+\kappa}
$$

and for the generation-time density function (up to a normalization factor)

$$
f(T)=\left\{\begin{array}{lc}
\int_{m(a,-T)}^{1 / 2} & (1-2 x)^{\kappa-1} x^{-\kappa-1} H(2 x) H(m(x, T)) \mathrm{d} x \\
& \ln [a /(1-a)] \leq r T \leq \ln [(2-a) /(1-a)] \\
\int_{a / 2}^{1 / 2}(1-2 x)^{\kappa-1} x^{-\kappa-1} H(2 x) H(m(x, T)) \mathrm{d} x & \ln [(2-a) /(1-a)] \leq r T<\infty
\end{array}\right.
$$

where

$$
H(x)=(x-a)^{2} \mathrm{e}^{\beta(x-a)} x^{-a^{2} \beta}(1-x)^{(1-a)^{2} \beta}
$$

and

$$
m(x, T)=\left\{1+[(1-x) / x] \mathrm{e}^{-r T}\right\}^{-1} .
$$

From equation (B2) we find that

$$
\beta(t)=2 \int_{a}^{1} \int_{m(x, t)}^{1} \phi_{b}(x) \phi_{b}(y) \mathrm{d} y \mathrm{~d} x,
$$

where $\phi_{b}(x)$ is given by (7).

To calculate $r_{s s}$ we must compute the first two moments for the distributions $f_{1 *}$ and $f_{2 *}$, given by (B5), and then calculate the correlation coefficient from the general expressions given in (B6). To calculate $r_{m d}$ we write equation (B7) as

$$
T_{1 d}+T_{2 m}=\frac{1}{r} \ln \left[\left(\frac{a}{1-a}\right) \mathrm{e}^{r T_{2 n i}+2}\right],
$$

which relates $T_{1 d}$ to $T_{2 m}$ for the case of logistic growth. Using this relation in equations (B8), we calculate numerically values for $\operatorname{Cov}\left(T_{m}, T_{d}\right), \operatorname{Var}\left(T_{m}\right)$ and $\operatorname{Var}\left(T_{d}\right)$, which determine $r_{m d}$ via (B9).

In Fig. 4 we compare the theoretical density functions (16) and (17) to the experimental histograms for fission yeast. The theoretical distributions are plotted 


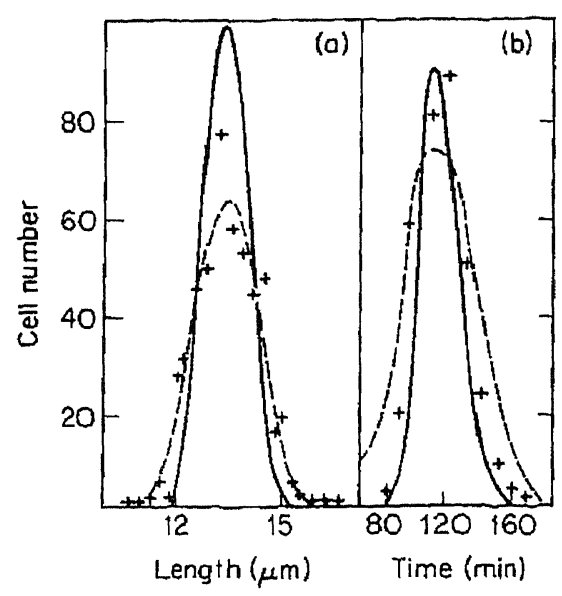

FIG. 4. Probability density functions for division size (a) and generation time (b), predicted by equations (16) and (17) and compared with the fission yeast data $(+)$ from Fig. 1. Parameter values for dashed curves: $a=0.6, \hat{b}=1.5 \mathrm{~min}^{-1}$; parameter values for solid curves: $a=0.6, \hat{b}=8.7 \mathrm{~min}^{-1}$. In both cases the specific growth rate, $k$ was chosen to agree with the observed mean generation time: $k \simeq$ $(\ln 2) /\langle T\rangle=0.693 / 1.16 \mathrm{~min}=0.006 \mathrm{~min}^{-1}$. These values of $a, \hat{b}$ and $k$ determine $r$ by way of the characteristic equation (14): $r=0.0143 \mathrm{~min}^{-1}$ for the dashed curves, $r=0.0124 \mathrm{~min}^{-1}$ for the solid curves. The normalization constants were chosen to give comparable overall numbers of cells.

for two different values of $\hat{b}$. Neither choice fits both the division size distribution and the generation time distribution exceptionally well. The smaller value $(\hat{b}=$ $1.5 \mathrm{~min}^{-1}$, dashed curve) fits the division size distribution well but not the generation time distribution, and vice versa for the larger value $\left(\hat{b}=8.7 \mathrm{~min}^{-1}\right.$, solid curve).

In Fig. 5 we compare the fission yeast data with the theoretical beta curve (18) for both values of $\hat{b}$. The larger value of $\hat{b}$ gives excellent agreement with experiment, but the smaller value is very much in disagreement with the experimental beta curve. Notice again that the shape of the beta curve is quite sensitive to $\beta=\hat{b} / r$, much more so than the shapes of $\phi(x)$ or $f(T)$.

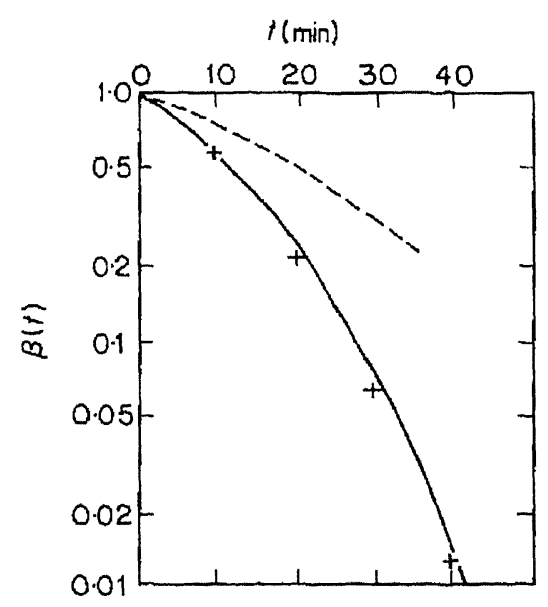

FIG. 5. Beta curves predicted by equation (18) and compared with the fission yeast data $(+)$. Parameter values: $\hat{b}=1.5 \mathrm{~min}^{-1}$ (dashed curve), $\hat{b}=8.7 \mathrm{~min}^{-1}$ (solid curve); in both cases $a=0.6$ and $k=$ $0.006 \mathrm{~min}^{-1}$. 
Finally we have calculated the correlation coefficients, $r_{s s}$ and $r_{\text {md }}$, as described above. For $\hat{b}=8.7 \mathrm{~min}^{-1}, a=0.6, k=0.006 \mathrm{~min}^{-1}, r=0.0124 \mathrm{~min}^{-1}$, we find that $r_{s s}=0.18$ and $r_{m d}=-0.39$. The predicted value of $r_{m d}$ does not differ significantly from the measured values $-0.44(n=48, P=70 \%)$; however, the predicted value of $r_{s s}$ is too far from the observed value for the differences to be attributed to chance. For the two experimental values of $r_{s s}$ reported earlier we find: $0.50(n=50, P=1 \%)$ and $0.39(n=36, P=20 \%)$ where $P$ is the probability that the difference between the predicted value $(0.18)$ and the measured value can be attributed to random sampling error on a sample of $n$ pairs.

Notice that, as might be expected, the correlation coefficients for logistic cell growth are less extreme (closer to zero) than the corresponding correlations for exponential cell growth. This feature of logistic growth is illustrated more generally in Fig. 6.

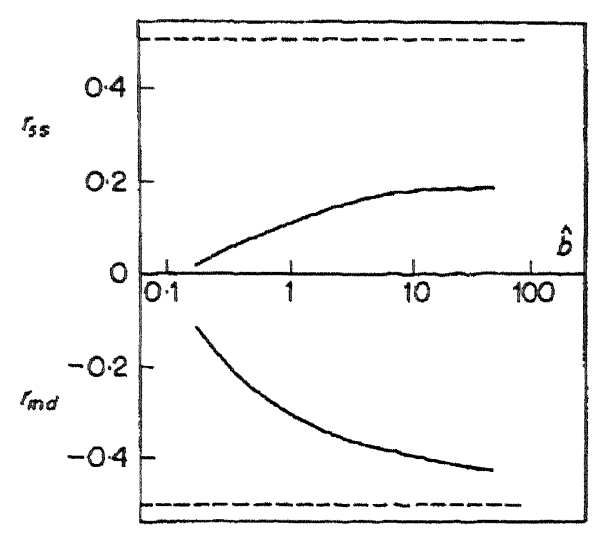

Fra. 6. Correlation coefficients for sloppy size control with logistic cell growth. Parameter values: $a=0.6, k=0.006, \hat{b}$ variable. The dashed lines indicate $r_{s s}$ and $r_{m d}$ for exponential cell growth.

\section{Discussion}

By "sloppy size control" we propose that the probability of cell division (per unit time) depends on present cell size and is independent of cell age, cell size at birth, or any other possible determinants. The size control mechanism that governs cell division is sloppy in the sense that cells do not all divide at the same size but rather the probability of division increases with size. We assume that no cells divide smaller than some constant size $X$ or larger than size $2 X$.

For a sloppy size control model to be well defined, we must specify the growth law governing individual cell growth and the function describing the division probability per unit time as a function of cell size. Given these two functions, we show how to compute (i) the probability density functions for division size and generation time, (ii) the correlation coefficients for generation times of sister-sister cell pairs and mother-daughter cell pairs, and (iii) the distribution function for the difference in generation times of sister cells (the so-called beta curve).

We have investigated two different growth laws: exponential growth and logistic growth. These two laws provide reasonable descriptions for the two major classes 
of cell growth: continuously accelerating or eventually decelerating (with respect to increasing cell size). The other function that needs to be specified, the division probability per unit time, is completely unknown from an experimental point-ofview. We have chosen for this function the simplest form that satisfies the basic assumptions of the model. This function can be estimated from time-lapse cinematographic records of proliferating cell cultures, but such estimates remain to be made.

To test theoretical models of the control of cell division it is necessary to have experimental measurements of a variety of descriptors of proliferating cell cultures. In general it is not sufficient to measure only, say, a histogram of generation times because such data do not contain sufficient information for a critical test of alternative theories. Generation time histograms are unimodal and described essentially by three numbers: the mean, variance and skewness of the distribution. Experience has shown that almost any reasonable model of cell growth and division, with three or more adjustable parameters, can be fitted to typical generation time histograms. However, if the generation time histogram is supplemented with data on generationtime correlations and/or division size distributions, then more rigorous assessments of theoretical models can be made.

The only extensive data set of which we are aware is the fission yeast data obtained by Miyata and his coworkers. Elsewhere Tyson (1985) has used the data in Fig. 1 to assess three widely divergent models of progress through the cell cycle: the transition probability model (Smith \& Martin, 1973), the tandem model (Tyson \& Hannsgen, 1985), and the sloppy size control model. He showed that neither the transition probability model nor the tandem model could give acceptable accounts of both the division size histogram and the generation time histogram. On the other hand, the sloppy size control model can fit both histograms.

In this paper we have extended the assessment of sloppy size control by comparing theoretical and experimental beta-curves and correlation coefficients as well as division size and generation time histograms. We find that the sloppy size control model, with only three adjustable parameters (the minimum size at division, the maximum size at division, and a first-order rate constant parameterizing the division probability function), can fit all the statistical data on fission yeast cultures.

The best fit of theory to experiment is obtained assuming that the size of individual yeast cells increases exponentially. If we assume logistic growth of individual cells, then we find that the theoretical division size histogram is considerably narrower than observed (see Fig. 4(a), solid curve) and the predicted correlation of sister cell generation times is significantly less positive than observed. Miyata has not reported any information on the growth law for individual cells under his culture conditions, though such information could be obtained from the time-lapse record of the culture. Mitchison \& Nurse (1985) report that large fission yeast cells near the end of the cell cycle show very little increase in cell length. This suggests that logistic growth may be more appropriate than exponential growth in describing the increase in cell length in fission yeast. So why is logistic growth less satisfactory than exponential growth within the sloppy size control model? Perhaps cell length is not the "size" variable that controls division probability. Cell "size" may be monitored by the cell itself in terms of the accumulation of some biochemical within each cell, and this 
variable may increase exponentially even though cell length increases non-exponentially. Alternatively, it may be that our particular choice for $b(x)$, the division probability function, is inappropriate. Perhaps a different choice of $b(x)$, combined with logistic growth, would perform as well as, or better than, our chosen $b(x)$ plus exponential growth.

This work was initiated while JJT was a Visiting Fellow at the Centre for Mathematical Biology, Oxford University, England, during Trinity term, 1984. JJT thanks Professor J. D. Murray for the opportunity to work to the Centre. We also are deeply grateful to Professor H. Miyata for supplying us with the experimental data used to calculate the beta curve and the generation-time correlation coefficients for the fission yeast culture. This work was supported in part by grants from the Science and Engineering Research Council (Great Britain) GR/C/63695 to the Centre for Mathematical Biology, the National Science Foundation (USA) MCS-8301104 to JJT and the National Institutes of Health (USA) GM-27629 to JJT.

\section{REFERENCES}

BeLl, G. I. \& Anderson, E. C. (1967). Biophys. J. 7, 329.

Brooks, R. F., BenNetT, D. C. \& SMrth, J. A. (1980). Cell 19, 493.

COLliNS, F. H. \& RICHMOND, M. H. (1962). J. gen. Microbiol. 28, 15.

Creanor, J. \& Mitchison, J. M. (1982). J. Cell Sci. 58, 263.

DANIEL, W. W. (1983). Biostatistics: A Foundation for Analysis in Health Sciences. p. 300. New York: Wiley.

Diekmann, O., Lauwerier, H. A., Aldenberg, T. \& Metz, J. A. J. (1983). J, math. Biol. $18,135$.

Diekmann, O., Heijmans, H. J. A. M. \& Thieme, H. R. (1984). J, math. Biol. 19, 227 (Part II is in preparation.)

Fantes, P. A., Grant, W. D., Pritchard, R. H., Sudbery, P. E. \& Wheals, A. E. (1975). J. theor. Biol. 50, 213.

FANTES, P. A. (1977). J. Cell Scl. 24, 51.

Heijmans, H. J. A. M. (1984). Math. Biosci. 72, 19.

John, P. C. L. (1981). The Cell Cycle. London: Cambridge Univ. Press.

KENDALL, D. (1948). Biometrika 35, 316.

KOCH, A. L. \& SCHAECHTER, M. (1962). J. gen. Microbiol. 29, 435.

KubitSCHEK, H. E. (1985). Preprint.

KUBITSCHEK, H. E. \& WARD, R. A. (1985). J. Bacteriol. 162, 902.

LAsotA, A. \& MACKEY, M. C. (1984). J. math. Biol. 19, 43.

Lebovitz, J. L. \& Rubinow, S. I. (1969), J. theor. Biol. 23, 99.

LORD, P. G. \& WheAls, A. E. (1981). J. Cell Sci. 50, 361.

METz, J. A. J. \& DIEkMANN, O. (eds) (in preparation). The Dynamics of Physiologically Structured Populations, Springer Lecture Notes in Biomathematics. Berlin, Heidelberg: Springer-Verlag.

MinoR, P. D. \& SMITH, J. A. (1974). Nature 248, 241.

Mitchison, J. M. (1957). Expl. Cell Res. 13, 244.

Mitchison, J. M. (1977). In: Mitosis: Facts and Questions (Little, M. et al. eds). p. 1. Berlin, Heidelberg: Springer-Verlag.

Mitchison, J. M. \& Nurse, P. (1985). J. Cell Sci. 75, 357.

Miyata, H., Mixata, M. \& Ito, M. (1978). Cell Struct. Funct. 3, 39.

NuRSE, P. (1980). Nature 286, 9.

Nurse, P. \& Streiblova, E. (1984). The Microbial Cell Cycle. Boca Raton, Florida: CRC Press.

Painter, P. R. \& MARR, A. G. (1968). Ann. Rev. Microbiol. 22, 519.

POWEll, E. O. (1964). J. gen. Microbiol. 37, 231.

RAHN, O. (1932). J. gen. Physiol. 15, 257.

Smith, J. A. \& MARTIN, L. (1973). Proc natn. Acad. Sci. U.S.A. 70, 1263.

Sudbery, P. E. \& GRANT, W. D. (1975). Expl. Cell Res. 95, 405.

TYSON, J. J. \& HANNSGeN, K. H. (1985). J. theor. Biol. 113, 29.

TYSON, J. J. (1985). BioEssays 2, 72.

Wheals, A. E. (1982). Mol. Cell. Biol. $2,361$. 


\section{APPENDIX A \\ Size Distributions and Generation Time Distributions}

If a population of cells is undergoing steady asynchronous exponential growth and division, then the conservation of cells of size $x$ implies that (Collins \& Richmond, 1962)

$$
\frac{\mathrm{d}}{\mathrm{d} x}[V(x) \lambda(x)]=k[2 \psi(x)-\phi(x)-\lambda(x)],
$$

where $k$ is the specific growth rate of the culture and $V(x)=$ growth rate of a cell of size $x, \phi(x)=$ probability density function for size at division in a sample of dividing cells, $\psi(\boldsymbol{x})=$ probability density function for size at birth in a sample of newborn cells, $\lambda(x)=$ probability density function for present size in a sample of extant cells.

We shall assume throughout that cells divide exactly in half, in which case

$$
\psi(x)=2 \phi(2 x)
$$

Furthermore, from the definition of $b(x)$, the division probability function, we have

$$
\begin{aligned}
b(x) \mathrm{d} t & =\frac{\# \text { cells of size } x \text { that divide between } t \text { and } t+\mathrm{d} t}{\# \text { cells of size } x \text { that are extant at time } t} \\
& =\frac{\phi(x) \cdot k N(t) \mathrm{d} t}{\lambda(x) N(t)}
\end{aligned}
$$

because $k N(t) \mathrm{d} t=$ total number of cells that divide between $t$ and $t+\mathrm{d} t$. Thus,

$$
k \phi(x)=b(x) \lambda(x)
$$

Substituting (A2) and (A3) in (A1), we obtain

$$
\frac{\mathrm{d}}{\mathrm{d} x}[V(x) \lambda(x)]=4 b(2 x) \lambda(2 x)-b(x) \lambda(x)-k \lambda(x) .
$$

Our assumption that $\frac{1}{2}<a<1$ implies that $\psi(x)$ and $\phi(x)$ do not overlap, i.e. that $\psi(x)=(2 / k) b(2 x) \lambda(2 x)=0$ for $\frac{1}{2}<x<1$, and that $\phi(x)=(1 / k) b(x) \lambda(x)=0$ for $a / 2<x<a$. With this information we can solve (A4) for $V(x) \lambda(x)$

$$
C V(x) \lambda(x)=\left\{\begin{array}{l}
\exp \left\{-\int_{a / 2}^{x}[k+b(\xi)][V(\xi)]^{-1} \mathrm{~d} \xi\right\}, \quad a<x<1, \\
\exp \left\{-\int_{a / 2}^{x} k[V(\xi)]^{-1} \mathrm{~d} \xi\right\}, \quad \frac{1}{2}<x<a, \\
\exp \left\{-\int_{a / 2}^{x} k[V(\xi)]^{-1} \mathrm{~d} \xi\right\} \int_{a / 2}^{x} 4 b(2 \xi) \\
\cdot[V(2 \xi)]^{-1} \exp \left\{-\int_{\xi}^{2 \xi}[k+b(\eta)][V(\eta)]^{-1} \mathrm{~d} \eta\right\} \mathrm{d} \xi, \quad a / 2<x<\frac{1}{2},
\end{array}\right.
$$

where $C$ is a normalization constant chosen so that $\int_{a / 2}^{1} \lambda(x) \mathrm{d} x=1$. 
We can express $\lambda(x)$ in a simpler form by introducing the following functions

$$
\begin{aligned}
& E(x)=\exp \left\{-\int_{a}^{x}[b(\xi) / V(\xi)] \mathrm{d} \xi\right\}, \\
& G(x)=\int_{a / 2}^{x}[V(\xi)]^{-1} \mathrm{~d} \xi, \\
& G_{2}(x)=G(2 x)-G(x)=\int_{x}^{2 x}[V(\xi)]^{-1} \mathrm{~d} \xi .
\end{aligned}
$$

$E(x)$ is the probability that a cell passing size $a$ will reach size $x$ before dividing. By assumption, $E(1)=0 . G(x)$ is the time needed for a cell to grow from size $a / 2$ (the minimum size at birth) to size $x . G_{2}(x)$ is the time needed for a cell presently of size $x$ to double its present size. In terms of these functions, our expression for $\lambda(x)$ becomes

$$
C V(x) \lambda(x)= \begin{cases}\mathrm{e}^{-k G(x)} E(x), \quad & a<x<1, \\ \mathrm{e}^{-k G(x)}, \quad \frac{1}{2}<x<a, & a / 2<x<\frac{1}{2} \\ \mathrm{e}^{-k G(x)} \int_{a / 2}^{x} K(\xi) \mathrm{e}^{-k G_{2}(\xi)} \mathrm{d} \xi, \quad\end{cases}
$$

where

$$
K(x)=4 b(2 x) E(2 x) / V(2 x)
$$

(In defining $K(x)$ we have made use of our assumptions that $b(x)=0$ for $x<a$ and that $a>\frac{1}{2}$.) The normalization constant is given by

$$
\begin{aligned}
C= & \int_{a}^{1}\left[\mathrm{e}^{-k G(x)} E(x) / V(x)\right] \mathrm{d} x+\int_{1 / 2}^{a}\left[\mathrm{e}^{-k G(x)} / V(x)\right] \mathrm{d} x \\
& +\int_{a / 2}^{1 / 2}\left[\mathrm{e}^{-k G(x)} / V(x)\right] \int_{a / 2}^{x} K(\xi) \mathrm{e}^{-k G_{2}(\xi)} \mathrm{d} \xi \mathrm{d} x .
\end{aligned}
$$

From equation (A3) we find, as well, that

$$
C=\frac{1}{k} \int_{a}^{1}\left[b(x) \mathrm{e}^{-k G(x)} E(x) / V(x)\right] \mathrm{d} x .
$$

The right-hand-sides of (A6) and (A7) are identical if and only if

$$
\int_{a / 2}^{1 / 2} K(\xi) \mathrm{e}^{-k G_{2}(\xi)} \mathrm{d} \xi=1 .
$$

(To prove this, interchange the order of integration in the double integral in (A6), after which all of the integrals except (A8) can be evaluated exactly by appropriate changes of variables.) Equation (A8) determines the specific growth rate of the culture as a whole in terms of the growth law for individual cells, $V(x)$, and the 
division probability function, $b(x)$. For instance, if cells grow exponentially, $V(x)=$ $r x$, then (A8) is satisfied for any $b(x)$ if and only if $k=r$, i.e. the specific growth rate of the culture is identical to the specific growth rate of the individual cells.

From equations (A3) and (A5) we have, for the division-size distribution defined on a sample of dividing cells

$$
k C \phi(x)=b(x) \mathrm{e}^{-k G(x)} E(x) / V(x), \quad a<x<1
$$

and for the birth-size distribution defined on a sample of newborn cells

$$
k C \psi(x)=2 b(2 x) \mathrm{e}^{-k \sigma(2 x)} E(2 x) / V(2 x), \quad a / 2<x<\frac{1}{2}
$$

The division-size distribution defined on a sample of newborn cells can be derived as follows. The cumulative distribution

$$
\Phi_{b}(x)=\int_{a}^{x} \phi_{b}(\xi) \mathrm{d} \xi
$$

is simply the probability that a newborn cell will divide before reaching size $x$. Since all newborn cells pass size $x=a$ at some time in their life, we have from the definition of $E(x)$ that $\Phi_{b}(x)=1-E(x)$, or

$$
\phi_{b}(x)=b(x) E(x) / V(x), \quad a<x<1 .
$$

The size distributions given by equations (A5), (A9), (A10) and (A11) are all defined in terms of contemporaneous samples of cells. If we sample cells by generation, then the "present size" distribution, $\lambda(x)$, is no longer meaningful, and the division size distribution is simply

$$
\phi_{*}(x)=b(x) E(x) / V(x), \quad a<x<1 .
$$

In general, $\phi_{b}$ and $\phi_{*}$ need not be identical, but they are so here because all newborn cells pass size $x=a$, whether they are drawn from a sample of contemporaneous newborns or from a sample of same-generation newborns.

Consider a sample of contemporaneous newborn cells. Let $x$ be the birth size and $\xi$ the division size of a cell from this sample. Then the generation time of such a cell must be $T=G(\xi)-G(x)$. Thus, the division size is given in terms of the birth size and generation time by $\xi=G^{-1}(T+G(x))$, where $G^{-1}$ is the inverse of $G($ i.e. $\left.G^{-1}(G(x))=x\right)$. It is convenient to let $m(x, t) \equiv G^{-1}(t+G(x))=$ size of cell at time $t$ if size $=x$ at time 0 . Now, the probability that the division size of a newborn cell lies between $\xi$ and $\xi+\mathrm{d} \xi$ is simply $\phi_{b}(\xi) \mathrm{d} \xi$. Thus, the probability that the generation time of a newborn cell of size $x$ lies between $T$ and $T+\mathrm{d} T$ is $\phi_{b}(m(x, T)) V(m(x, T)) \mathrm{d} T$, since $\mathrm{d} \xi=V(\xi) \mathrm{d} T$. Summing up these conditional probabilities over all the newborn cells in the sample, we find that

$$
f(T)=\int_{a / 2}^{1 / 2} \psi(x) \phi_{b}(m(x, T)) V(m(x, T)) \mathrm{d} x, \quad T_{\min }<T<T_{\max }
$$

where $T_{\min }=G(a)-G\left(\frac{1}{2}\right), T_{\max }=G(1)$.

In (A13) $\psi(x)$ is to be given by (A10) and $\phi_{b}(x)$ by (A11). 
If we desire the generation time distribution of a sample of same-generation cells, then a similar argument yields

$$
f_{*}(T)=\int_{a / 2}^{1 / 2} 2 \phi_{*}(2 x) \phi_{*}(m(x, T)) V(m(x, T)) \mathrm{d} x, \quad T_{\min }<T<T_{\max }
$$

where $\phi_{*}(x)$ is given by (A12).

Equation (A14) is identical to Powell's equation (29) (Powell, 1964, p. 242). To see this, notice that Powell's frequency function for size at termination, $l\left(x_{t}\right)$, is identical with our function $\phi_{b}(x)$, which is also the same as $\phi_{*}(x)$ as long as $a>\frac{1}{2}$. Furthermore, Powell assumes exponential growth, $V(x)=\nu x(\nu=$ specific growth rate) and unequal fission; whereas equation (A14) is written down for a general growth law and exactly equal fission. Powell's frequency function, $k(p)$, we assume to be a delta "function" at $p=\frac{1}{2}$. Notice that Powell never clearly distinguishes between contemporaneous sampling of cells and generation-by-generation sampling. However, it is obvious from his derivation that the function $f(\tau)$ in his equation (29) is generation-based, i.e. what we have derfoted by $f_{*}(T)$. Powell's "carrier distribution", $C(\tau)$ on p. 245 , is the distribution we would call $f_{m}(T)$.

Equation (A14) is also consistent with an equation given by Lasota \& Mackey $(1984$, p. 46) for the density function for the distribution of generation times. Our equations can be compared to theirs by noting that their function $\alpha(r, t)$ is identical with our $E(m(r, t))$, and their $f(r)$ is our $\psi_{*}(r)$.

\section{APPENDIX B}

\section{Beta Curve and Correlation Coefficients}

For the sloppy size control model we can calculate $\beta(t)$ as follows. Let us split the generation time of a cell into two components, $T=T_{1}+T_{2}$, where $T_{1}$ is the time taken to grow from birth to size $a$, the minimum size at division, and $\boldsymbol{T}_{2}$ is the time spent from reaching size $a$ until cell division. Sister cells, which are assumed to be identical in size at birth, have identical values for $T_{1}$ and differ only in $T_{2}$. Let $f_{2}\left(T_{2}\right)$ be the probability density for $T_{2}$ in a sample of newborn cells. Suppose that the first sister to divide divides at $T_{2}=s$. Then

$$
\beta(t)=2 \int_{0}^{T_{2, \max }} P(s, t) f_{2}(s) \mathrm{d} s
$$

where $T_{2, \max }=G(1)-G(a), P(s, t)=$ probability that a cell is still undivided at time $s+t$ after reaching size $a$, and the factor of 2 arises since either sister of a pair may divide first. Since the size of a cell at time $s+t$ after reaching size $a$ is $m(a, s+t)$, the probability that such a cell is still undivided at this size is $\int_{m(a, s+t)}^{T} \phi_{b}(x) \mathrm{d} x$. Thus

$$
\beta(t)=2 \int_{0}^{T_{2, \max }} f_{2}(s) \int_{m(a, s+t)}^{x} \phi_{b}(x) \mathrm{d} x \mathrm{~d} s .
$$


To determine $f_{2}(\cdot)$, notice that

$$
\begin{aligned}
\int_{0}^{t} f_{2}(s) \mathrm{d} s & =\text { Prob }\{a \leq \text { division size } \leq m(a, t)\} \\
& =\int_{a}^{m(a, t)} \phi_{b}(x) \mathrm{d} x .
\end{aligned}
$$

Thus

$$
f_{2}(t)=\phi_{b}(m(a, t)) V(m(a, t))
$$

So, we have

$$
\beta(t)=2 \int_{0}^{T_{2, \max }} \int_{m(a, s+t)}^{1} \phi_{b}(x) \phi_{b}(m(a, s)) V(m(a, s)) \mathrm{d} x \mathrm{~d} s .
$$

We can put this equation in neater form by changing the variable of integration from $s$ to $r=m(a, s)$

$$
\beta(t)=2 \int_{a}^{m(1,-t)} \int_{m(r, t)}^{1} \phi_{b}(r) \phi_{b}(x) \mathrm{d} x \mathrm{~d} r .
$$

Notice that $\beta(0)=1$ and $\beta\left(T_{2, \text { max }}\right)=0$. For the case of exponential growth of individual cells,

$$
\beta(t)=2 \int_{a}^{e^{-k t}} \int_{r e^{k t}}^{l} \phi_{b}(r) \phi_{b}(x) d x d r .
$$

To get product moment correlation coefficients for sister-sister pairs and motherdaughter pairs, we need to know the probability density, call it $f_{1}(\cdot)$, for $T_{1}$ in a sample of newborn cells (where $T_{1}$ is the time necessary to grow from birth to size a). Notice that $T_{1, \min }=G(a)-G\left(\frac{1}{2}\right)$ and $T_{1, \max }=G(a)$. Now

$$
\begin{aligned}
\int_{T_{1, \min }}^{t} f_{1}(s) \mathrm{d} s & =\operatorname{Prob}\left\{T_{1, \min } \leq \mathrm{T}_{1} \leq \mathrm{t}\right\} \\
& =\operatorname{Prob}\left\{m(a,-t) \leq \text { birth size } \leq \frac{1}{2}\right\} \\
& =\int_{m(a,-t)}^{1 / 2} \psi(x) \mathrm{d} x
\end{aligned}
$$

Since $\psi(x)=2 \phi(2 x)$, we have

$$
f_{\mathrm{l}}(t)=2 \phi(2 m(a,-t)) V(m(a,-t)) .
$$

To get product moment correlation coefficients for generation times defined on a contemporaneous sample of newborn cells is difficult because $f_{1}(\cdot)$ is defined in terms of $\phi(x)$ and $f_{2}(\cdot)$ in terms of $\phi_{b}(x)$. If we choose to correlate generation times based on same-generation samples, then (B1) and (B4) become

$$
\begin{aligned}
& f_{1 *}(t)=2 \phi_{*}(2 m(a,-t)) V(m(a,-t)), \\
& f_{2 *}(t)=\phi_{*}(m(a, t)) V(m(a, t)),
\end{aligned}
$$

where $\phi_{*}(x)$ is given by (A12). 
Let $T_{a}$ and $T_{b}$ be the generation times of two sister cells. Then, by definition,

$$
r_{s s}=\frac{\operatorname{Cov}\left(T_{a}, T_{b}\right)}{\left[\operatorname{Var}\left(T_{a}\right) \operatorname{Var}\left(T_{b}\right)\right]^{1 / 2}}
$$

Because sister cells have identical $T_{1}$ 's, $T_{a}=T_{1}+T_{2 a}$ and $T_{b}=T_{1}+T_{2 b}$. In this case

$$
\operatorname{Var}\left(T_{a}\right)=\operatorname{Var}\left(T_{b}\right)=\operatorname{Var}\left(T_{1}\right)+\operatorname{Var}\left(T_{2}\right)
$$

and

$$
\operatorname{Cov}\left(T_{a}, T_{b}\right)=\operatorname{Var}\left(T_{1}\right)
$$

since $T_{1}, T_{2 a}$ and $T_{2 b}$ are all uncorrelated random variables. Thus

$$
r_{s s}=\frac{\operatorname{Var}\left(T_{1}\right)}{\operatorname{Var}\left(T_{1}\right)+\operatorname{Var}\left(T_{2}\right)}
$$

Let $T_{m}$ and $T_{d}$ be the generation times of mother and daughter cells, with $T_{m}=T_{1 m}+T_{2 m}$ and $T_{d}=T_{1 d}+T_{2 d}$. Let $2 X$ be the size of the mother cell at division. Then $T_{2 m}=G(2 X)-G(a), T_{1 d}=G(a)-G(X)$, and $T_{2 m}+T_{1 d}=G_{2}(X)$ where $G_{2}(X)$ is the time necessary to grow from size $X$ to size $2 X$. Because $2 X=$ $G^{-1}\left(T_{2 m}+G(a)\right)$, we have

$$
T_{1 d}=G_{2}\left(m\left(a, T_{2 m}\right) / 2\right)-T_{2 m}
$$

Equation (B7) relates $T_{1 d}$ to $T_{2 m}$. Except for this relation, the times $T_{1 m}, T_{2 m}, T_{1 d}$ $T_{2 d}$ are uncorrelated random variables. Thus, letting $\bar{T}_{1}$ and $\bar{T}_{2}$ represent the mean times in phases 1 and 2 of the cell cycle, we can write

$$
\begin{aligned}
\operatorname{Cov}\left(T_{m}, T_{d}\right) & =E\left[\left(T_{2 m}-\bar{T}_{2}\right)\left(T_{1 d}-\bar{T}_{1}\right)\right] \\
\operatorname{Var}\left(T_{m}\right) & =\operatorname{Var}\left(T_{1}\right)+\operatorname{Var}\left(T_{2}\right) \\
\operatorname{Var}\left(T_{d}\right) & =E\left[\left(T_{1 d}-\bar{T}_{1}\right)^{2}\right]+\operatorname{Var}\left(T_{2}\right)
\end{aligned}
$$

where the expectations are taken over the distribution $f_{2 *}\left(T_{2 m}\right)$ and $T_{1 d}$ is given as a function of $T_{2 m}$ by (B7). From these numbers we then calculate

$$
r_{m d}=\frac{\operatorname{Cov}\left(T_{m}, T_{d}\right)}{\left[\operatorname{Var}\left(T_{m}\right) \operatorname{Var}\left(T_{d}\right)\right]^{1 / 2}} .
$$

For the special case of exponential growth, $V(x)=k x$, we have

$$
\begin{aligned}
& f_{1 *}(t)=2 k a \mathrm{e}^{-k t} \phi_{*}\left(2 a \mathrm{e}^{-k t}\right), \\
& f_{2 *}(t)=k a \mathrm{e}^{k t} \phi_{*}\left(a \mathrm{e}^{k t}\right) .
\end{aligned}
$$

Notice that, convolving $f_{1 *}$ with $f_{2 *}$, we obtain $f_{*}(T)$, as we should. Calculating the moments of $T_{1}$ and $T_{2}$, we find that

where $\tau=(\ln 2) / k$, and

$$
E\left(T_{2}\right)=\tau-E\left(T_{1}\right)
$$

$$
E\left(T_{2}^{2}\right)=\tau^{2}-2 \tau E\left(T_{1}\right)+E\left(T_{1}^{2}\right)
$$


Thus,

$$
\operatorname{Var}\left(T_{2}\right)=E\left(T_{2}^{2}\right)-\left(E\left(T_{2}\right)\right)^{2}=E\left(T_{1}^{2}\right)-\left(E\left(T_{1}\right)\right)^{2}=\operatorname{Var}\left(T_{1}\right)
$$

Thus, from equation (B6) we have $r_{s s}=+\frac{1}{2}$, if cells grow exponentially and divide accurately in half.

For mother and daughter cells that grow exponentially and divide accurately in half, we have from equation (B7) $T_{1 d}=\tau-T_{2 m}$ where $\tau=(\ln 2) / k$. Thus, $\operatorname{Cov}\left(T_{m}, T_{d}\right)=-\operatorname{Var}\left(T_{2}\right), \operatorname{Var}\left(T_{m}\right)=\operatorname{Var}\left(T_{1}\right)+\operatorname{Var}\left(T_{2}\right)$, and $\operatorname{Var}\left(T_{d}\right)=2 \operatorname{Var}\left(T_{2}\right)$. Now $\operatorname{Var}\left(T_{1}\right)=\operatorname{Var}\left(T_{2}\right)$, so $r_{m d}=-\frac{1}{2}$, if cells grow exponentially and divide exactly in half.

With characteristic elegance Powell (1964) showed that, if cells grow exponentially but divide inaccurately, then $r_{s s}$ is somewhat smaller than $+\frac{1}{2}, r_{m d}$ is somewhat larger than $-\frac{1}{2}$, and $r_{s s}+r_{m d}<0$. 